Nonlin. Processes Geophys., 25, 511-519, 2018

https://doi.org/10.5194/npg-25-511-2018

(C) Author(s) 2018. This work is distributed under

the Creative Commons Attribution 4.0 License.

\title{
Exceedance frequency of appearance of the extreme internal waves in the World Ocean
}

\author{
Tatyana Talipova $^{1,2}$, Efim Pelinovsky ${ }^{1,2}$, Oxana Kurkina ${ }^{1}$, Ayrat Giniyatullin ${ }^{1}$, and Andrey Kurkin ${ }^{1}$ \\ ${ }^{1}$ Nizhny Novgorod State Technical University n.a. R.E. Alekseev, Nizhny Novgorod, Russia \\ ${ }^{2}$ Institute of Applied Physics, Nizhny Novgorod, Russia
}

Correspondence: Andrey Kurkin (aakurkin@gmail.com)

Received: 6 February 2018 - Discussion started: 12 February 2018

Revised: 5 July 2018 - Accepted: 6 July 2018 - Published: 18 July 2018

\begin{abstract}
Statistical estimates of internal waves in different regions of the World Ocean are discussed. It is found that the observed exceedance probability of large-amplitude internal waves in most cases can be described by the Poisson law, which is one of the typical laws of extreme statistics. Detailed analysis of the statistical properties of internal waves in several regions of the World Ocean has been performed: tropical part of the Atlantic Ocean, northwestern shelf of Australia, the Mediterranean Sea near the Egyptian coast, and the Yellow Sea.
\end{abstract}

\section{Introduction}

Internal waves are observed everywhere in the shelf zones of seas. The main source of their generation in the ocean is the semidiurnal tidal wave, which is initially barotropic and generates the baroclinic tidal wave by scattering on the continental shelf. Periodical lunar tide M2 generates internal waves with a period of $12.4 \mathrm{~h}$. This process is well studied and presented in publications (Garret and Kunze, 2007; Morozov, 1995, 2018; Vlasenko et al., 2005). Nevertheless the variability of the magnitude of the lunar tide and variations in the temperature and salinity of the sea water lead to random characteristics of the observed internal wave field; see, for example, the book by Miropolsky (2001) and review paper by Helfrich and Melville (2006). Spectral and correlation methods of random internal wave field are widely applied in science. As a result, climatic spectra of internal waves have been determined. The well-known model of Garret and Munk (1975) became the basis for the background spectra of internal waves in the World Ocean. This model determines the background of oceanic internal wave spectra, over which intense processes of internal wave generation occur leading to the appearance of large-amplitude (up to extreme values of $500 \mathrm{~m}$ ) internal waves (Alford et al., 2015). Data of the largeamplitude internal waves in various areas of the World Ocean are collected in numerous papers (Apel et al., 1985; Salusti et al., 1989; Holloway et al., 1999; Morozov, 1995, 2018; Ramp et al., 2004; Sabinin and Serebryany, 2007; Shroyer et al., 2011; Xu and Yin, 2012; Kozlov et al., 2014; Xu et al., 2016). For example, extreme waves of high amplitudes in the Strait of Gibraltar and Kara Gates Strait were analyzed in Morozov et al. (2002, 2003, 2008). Large-amplitude internal waves are of interest to researchers due to their dangerous impact on offshore platforms (Fraser, 1999; Song et al., 2011), their influence on safety of submarines and underwater vehicles (Osborn, 2010), and the fact that they also cause phase fluctuations of acoustic signals over large distances (WarnVarnas et al., 2003; Rutenko, 2010; Si et al., 2012). Special warning systems are developed now in regions of high risk of a pipe and platform damage by intense internal waves (Stöber and Moum, 2011).

Internal waves in the ocean can be considered as a continuous random process, and their intense large amplitudes can be interpreted as outliers of a random process and be described by the tails of the distribution functions. Consequently, the statistics of these processes is usually different from the Gaussian (normal) distribution. Non-Gaussian character of the observed internal wave field has been reported in many regions of the World Ocean (Miropol'sky, 2001; Wang and Gao, 2002). Seasonal and longitudinal statistical analysis of internal wave field has been reported recently in the South China Sea (Zheng et al., 2007). It is demonstrated that 
the largest number of internal wave packets is observed at a longitude of $116.5^{\circ} \mathrm{E}$ (in latitudinal band $20-22^{\circ} \mathrm{N}$ ). It has been reported that the most intense packets of internal waves in the South China Sea are generated in June. Special analysis of wave amplitude distribution for the tropical part of the western Atlantic, over the northwestern shelf of Australia and in the eastern Mediterranean Sea was performed in Ivanov et al. (1993a, b) and Pelinovsky et al. (1995). The authors show that the Poisson law is a good approximation for amplitude distributions of such waves. It is known (Gumbel, 1958; Leadbetter et al., 1983) that the Poisson distribution is widely used in the extreme statistics and very often applied in the ocean engineering for describing storm waves (Pelinovsky and Kharif, 2016), tsunamis (Kaistrenko, 2014) and rogue waves (Kharif et al., 2009); it is also used in geophysics for the description of climatic anomalies (Dischel, 2002). However, the distribution law for temperature deviations in the tropical part of the eastern Atlantic (Mesopolygon-85) is closer to the Gaussian distribution (Morozov et al., 1998), which can be explained by lower energy of internal waves in this region.

In this paper we apply the methods of extreme statistics to large-amplitude internal waves and present a brief review. First, the theoretical approach is revised in Sect. 2. Then, the results of statistical processing of the internal wave records in various regions of the World Ocean are presented in Sect. 3. Conclusion is given in Sect. 4.

\section{Extreme statistics methods for high-amplitude internal waves}

Let $\eta(t, \mid x, y, z)$ describe the vertical displacement of any isopycnal surface at fixed point which, in the first approximation, can be considered as stationary random process. Usually, a few central moments

$\mu_{r}=\int_{-\infty}^{\infty}(\eta-\bar{\eta})^{r} P(\eta) \mathrm{d} \eta(r=2,3,4)$

are computed for the statistical analysis. Here $P(\eta)$ is the probability density function and $\bar{\eta}$ is the mean value $\bar{\eta}=$ $\int_{-\infty}^{\infty} \eta P(\eta) \mathrm{d} \eta$ (unperturbed position of isopycnal surface).

The second moment $\mu_{2}=\sigma^{2}$ determines the intensity of internal wave oscillations and $\sigma$ is root mean square height of internal waves. The third and fourth moments determine skewness $\mathrm{Sk}=\mu_{3} / \sigma^{3}$ and kurtosis $\mathrm{Ku}=\mu_{4} / \sigma^{4}$, which are used to characterize the deviation of the distribution function from the Gaussian law (note that $\mathrm{Sk}=0$ and $\mathrm{Ku}=3$ for the Gaussian distribution). The sign of the skewness on our opinion can be explained by the specific shape in the internal Stokes wave, which is followed from the weakly nonlinear theory of internal waves. Unlike nonlinear surface waves, which always have narrow and high crests and flat troughs, internal waves at different depths can have either narrow crests and flat troughs or vice versa depending on the density stratification and modal structure, and distance to the bottom or surface. In the case of energetic internal waves of the first mode, the character of asymmetry of wave profile with respect to the horizontal axis is determined by the coefficient of the quadratic nonlinear term in the Korteweg-de Vries equation, which is strongly variable in the World Ocean (Grimshaw et al., 2007; Kurkina et al., 2011, 2017a, b). Computations of the skewness and kurtosis as well as distribution function of nonlinear internal waves are interesting problems that have been poorly studied to date.

Here we will use the direct method to evaluate the statistics of large-amplitude internal waves. We fix the reference vertical displacement and analyze the statistics of the exceedance of wave oscillations beyond this level (outliers of random process). Let us briefly reproduce the well-known approach for calculating the exceedance frequency for continuous processes (Gumbel, 1958; Stuart, 2001) with application to internal waves.

It is known from the vertical structure of internal waves that the largest amplitudes of the most energetic lowest-mode waves are found in the pycnocline. For definiteness, we chose the vertical displacement in the pycnocline and denote it $\eta(t)$ omitting coordinates of the pycnocline in this function. Both conditions for the outlier beyond the level $A$ in the interval $\Delta t$ should be satisfied at once:

$\eta(t)<A$ and $\eta(t+\Delta t)>A$.

Due to the smallness of $\Delta \mathrm{t}$ we can assume that $\eta(t+\Delta t) \approx$ $\eta(t)+w(t) \Delta t$, where $w(t)=\mathrm{d} \eta / \mathrm{d} t>0$ is the vertical velocity of water particles located within the isopycnal surface, and rewrite condition (2) as

$A-w(t) \Delta t<\eta(t)<A$.

The required probability of finding $\eta(t)$ in the interval (3) is

$P(A-w \Delta t<\eta<A)=\int_{0}^{\infty} \mathrm{d} W \int_{A-w \Delta t}^{A} f(\eta, W ; t) \mathrm{d} \eta$,

where $f(\eta, w ; t)$ is the two-dimensional probability density of vertical displacement $\eta(t)$ and vertical velocity $w(t)$ at the same time moment and the same coordinates. Since $\Delta t$ is small, we can use the mean-value theorem to calculate the inner integral in Eq. (4) and write

$P(\eta-w \Delta t<\eta<A)=\Delta t \int_{0}^{\infty} w f(A, w ; t) \mathrm{d} w$.

Probability density function (in time) can be easily found from Eq. (5):

$p(A ; t)=\int_{0}^{\infty} w f(A, w ; t) \mathrm{d} w$. 
Similarly, the probability of crossing level $A$ from the top down (into the region of small isopycnal value displacement) is

$p^{\prime}(A ; t)=-\int_{-\infty}^{0} w f(A, w ; t) \mathrm{d} w$,

since this requires $w<0$. This equation can be used to calculate the probability of large troughs in the vertical displacement, which can be as dangerous as large crests. It is known that polarity of large internal waves in the ocean is usually negative; this correlates with the negative sign of the quadratic nonlinearity parameter in the weakly nonlinear theory based on the Korteweg-de Vries equation for the deepest parts of the World Ocean (Grimshaw et al., 2007).

Here we calculate the average number of "positive" outliers (large crests) in the wave record. To do so we divide the total time interval into small subintervals $\Delta t_{j}$ and introduce a random value $N_{j}$ equal to 1 for an outlier and 0 outside the outlier. Then the total number of outliers is $N(A)=\sum N_{j}$ and its mean value is the ensemble average, the probability of this is equal to the probability of crossing level (6). Moving on to the limit for $\Delta t_{j} \rightarrow 0$, we finally have

$<N(A)>=\int_{0}^{T} \int_{0}^{\infty} w f(A, w ; t) \mathrm{d} w \mathrm{~d} t$.

In the case of a stationary random process formula (8) is simplified to

$<N(A)>=T \int_{0}^{\infty} w f(A, w) \mathrm{d} w$.

Thus, the average number of outliers is proportional to the time interval and falls with the increase in the outlier level. The same approach can be used to compute the average number of "negative" outliers (deepest troughs) in the internal wave field.

Only the average number of outliers was discussed above without considering their probabilistic distribution. A much more difficult problem is to calculate the latter. It should be noted that if outliers are rather rare (which is typical for very large-amplitude internal waves, $A \rightarrow \infty$ ), then their distribution can be regarded as the Poisson law. Then the probability that at least one outlier appears in the time interval $t$ is

$P=1-\exp (-v t)$

where the mean frequency of outliers $\nu=\langle N>/ T$ is found from Eq. (9) as

$$
v(A)=\int_{0}^{\infty} w f(A, w) \mathrm{d} w .
$$

The average frequency of outliers in the first approximation can be used as an estimate of the internal wave exceedance (cumulative) frequency with the amplitudes greater than the given value of $A$.

Detailed calculations of the outlier characteristics in the internal wave field require the knowledge of two-point (vertical displacement and vertical velocity) distribution functions of isopycnal variation, which are usually not measured. If the internal wave random field is assumed normal, the density of distribution function is described by the Gaussian law:

$f(\eta)=\frac{1}{\sqrt{2 \pi} \delta} e^{-\frac{(\eta-\bar{\eta})^{2}}{2 \delta^{2}}}$,

where $\delta$ is the standard deviation (mean amplitude of internal waves). The distribution function of the vertical displacement and the vertical velocity for the normal process do not correlate; hence their two-dimensional probability density splits into a product of two Gaussian curves (Eq. 12), which naturally have different mean-square deviations. Then the average frequency of outliers can be written as

$v(A)=\frac{\delta_{\mathrm{w}}}{2 \pi \delta_{\eta}} \exp \left(-\frac{A^{2}}{2 \delta_{\eta}^{2}}\right)$,

where $\delta_{\mathrm{w}}$ is the mean-square (standard deviation) value of the vertical velocity in the internal wave and $\delta_{\eta}$ is the meansquare value of the vertical isopycnal displacement. Thus, the internal wave exceedance frequency depends on the wave amplitude according to the Gaussian law, which very quickly decreased with an increase in amplitude. We will demonstrate the Gaussian character of cumulative frequency for tropical zone of the eastern Atlantic Ocean.

It should be remembered that usually the statistical distributions of internal wave field in various regions of the World Ocean are different from the normal distribution as we have already pointed out in the Introduction (see the book by Miropolsky, 2001); hence, the result will be different from Eq. (13) depending on the particular form of the tails of the distribution function in the large amplitude range. As shown in Leadbetter et al. (1983), the intermediate asymptotic exceedance frequency for large outliers is described by the Poisson law:

$v=v_{0} \exp \left(-\frac{A}{A_{0}}\right)$,

where $v_{0}$ and $A_{0}$ are the parameters depending on the specific type of "tails" of the distribution function in the large amplitude range. This expression can be used to compute exceedance (cumulative) frequency of large outliers (positive or negative) in the internal wave field. Obviously, the predicted amplitude values are also random, and here one can speak only about its evaluation. Thus to estimate the predicted amplitude $A$ it is necessary to set the value of the exceedance frequency:

$v=1 / T$, 
where $T$ is the prediction time (or the recording time). Expression (14) is used to calculate amplitude $A$ of internal wave over prognostic time interval $T$ :

$A_{T}=A_{0} \ln \left(\nu_{0} T\right)$.

Characteristic values of $A_{0}$ and $v_{0}$ are different in various regions of the World Ocean, and we shall discuss this in the next section.

\section{Statistics of internal wave field}

\subsection{Probability density function in the Yellow Sea}

Data analysis of measurements in the shallow water (Qingdao offshore area) of the Yellow Sea is reported in Wang and Gao (2002). The authors used a thermistor chain. The duration of records is $49 \mathrm{~h} 49 \mathrm{~min}$ with a sampling interval of $6.4 \mathrm{~s}$. The water depth is $33 \mathrm{~m}$. A thin unperturbed pycnocline is located in the interval from 10 to $16 \mathrm{~m}$ with the maximum of the Brunt-Väisälä frequency $N_{\max }=0.067 \mathrm{~s}^{-1}$. Vertical displacements (double amplitudes!) of high-pass-filtered 25$17.5^{\circ}$ (16 levels) isotherms are shown and their histograms are plotted. The maximum wave height here did not exceed $5 \mathrm{~m}$; nevertheless the process differs from the Gaussian process. It is found that the standard deviation increases slowly from 0.46 to $0.56 \mathrm{~m}$ from the surface to the bottom. Skewness is negative for each isotherm and its maximum absolute value is 0.5 in absolute value at a depth of $15 \mathrm{~m}$; it decreases to 0.36 at the surface and to 0.06 close to bottom. Kurtosis changes from 3.24 close to bottom to 5.05 at the $14 \mathrm{~m}$ of depth and 4.12 near the surface. It is shown that the distribution of large internal wave amplitudes does not coincide with the Gaussian distribution.

We can explain the sign of the computed skewness applying the weakly nonlinear theory of internal waves based on the Korteweg-de Vries equation (Pelinovsky and Shurgalina, 2017). In this region of the Yellow Sea the sign of quadratic nonlinear term in this equation is negative because the water stratification (see Wang and Gao, 2002) is practically approximated by a two-layer with pycnocline located above the mid-depth (Djorjevich and Redekopp, 1978; Kakutani and Yamasaki, 1978). Nonlinear waves as solutions of the extended Korteweg-de Vries equation with negative quadratic nonlinearity have deepest troughs. For instance, internal wave soliton has negative polarity (Grimshaw et al., 2007). This leads to the negative values of skewness.

\subsection{Exceedance frequency of internal waves in the tropical zone of the western Atlantic}

The exceedance frequency of internal wave is estimated using the data obtained during the 39th cruise of the RV Akademik Vernadsky in the northwestern Atlantic tropical zone near the mouth of the Amazon River $\left(2-15^{\circ} \mathrm{N}, 38\right.$ $52^{\circ} \mathrm{W}$; Ivanov et al., 1993b). Internal waves of moderate
$(2.5-10 \mathrm{~m})$ amplitudes are observed in this region. The ship echo sounder was used to obtain long-term internal wave records. This device allowed the authors to study the fluctuations of the sound-scattering layer at depths up to $100 \mathrm{~m}$. It is known that fluctuations of this layer can be caused by various processes, but in the range of periods up to $3 \mathrm{~h}$ they are mainly related to internal waves. The wave period on the sonar records varies in a wide range from 3 to $30 \mathrm{~min}$ on the sonar recordings. Since the measurements were made from the ship moving at a velocity of about $V=15 \mathrm{kn}$ and as the maximum of internal wave speed is $c=3 \mathrm{kn}$, in the first approximation, the internal wave pattern can be considered frozen in the first approximation. In this case, the "true" wave period increases in comparison to the observed one with a ratio of $V / c=5$. The amplitude of sound-scattering layer fluctuations was associated with the internal wave amplitude in the pycnocline everywhere. The total record duration was about $218 \mathrm{~h}$. Wave height (defined as the fluctuation swing between adjacent extremes) and fluctuation duration of the recording were considered the main characteristics. Primary echogram processing results by day are presented in Ivanov et al. (1993b). These data are used to estimate the exceedance (cumulative) frequency. They well agree with the regression line

$v=9.2 \exp (-0.3 H)$,

where $H$ is the wave height measured in meters, and the dimension of $v$ is in $\mathrm{h}^{-1}$, except for the heights greater than $25 \mathrm{~m}$, where the total number of wave observations does not exceed six. Expression (17) is used to estimate the predicted wave height versus predicted time function

$H=18+3.3 \ln T$.

Predicted values of internal wave heights versus time are summarized in Table 1.

During the time of measurements in the region, the "true" internal wave recording time (considering the ship motion) was about 45 days. According to the prediction for this period, a wave with a height of more than $31 \mathrm{~m}$ should be observed once, with a height of more than $23 \mathrm{~m}$ - twice, and more than $27 \mathrm{~m}$ - three times. In fact, the level of $31 \mathrm{~m}$ was exceeded three times, and the level of 27-28 $\mathrm{m}$ was exceeded six times, which indicates that a good agreement exists between the measurements and the predictive models.

\subsection{Temperature fluctuations caused by internal waves at Mesopolygon-85 in the Atlantic Ocean}

It is expected that the wave processes in the open ocean are described by the normal law, which makes it possible to use the theory of normal random process and estimate the limits of its applicability for internal waves. In this paper exceedance frequency analysis is undertaken for internal wave records obtained from a cluster of moorings 
Table 1. Wave height prediction for the Atlantic tropical zone.

\begin{tabular}{rrrrrrr}
\hline Time & 1 day & 10 days & 1 month & 3 months & 6 month & 1 year \\
\hline$H(\mathrm{~m})$ & 18 & 26 & 29 & 33 & 35 & 38 \\
\hline
\end{tabular}

in the eastern Atlantic Ocean in 1985 (the Mesopolygon85 experiment; the detailed description of the experiment is given in Kort (1988). Seventy-six moorings with current and temperature meters were deployed in the study area called Mesopolygon- 85 in the eastern part of the Atlantic Ocean with the objective of studying mesoscale variability of hydrophysical processes. The study site was located between the Canary Basin and the Cabo Verde Basin $\left(19-21^{\circ} \mathrm{N}\right.$ and $36-38^{\circ} \mathrm{E}$ ). The moorings operated approximately two months from April to May. The instruments were set at four levels, but the most representative measurements were gathered at the height level of $200 \mathrm{~m}$. The total size of the study site was approximately 148.2 by $148.2 \mathrm{~km}$. The sampling interval was $15 \mathrm{~min}$. In the Mesopolygon area, the bottom is covered with hills from 500 to $1000 \mathrm{~m}$ high over the floor. Such hills are located every 10 or 20 miles. They form a corrugated bottom topography over which the horizontal streamlines of barotropic currents are deformed. Thus, the internal tide is generated immediately in this area over the deepsea bottom topography. It should be mentioned that there 49 buoy records available in the region, and it is a unique possibility to estimate the horizontal variability of the internal wave amplitude distribution function.

Records of temperature variations (centigrade) at various points of the study site were used to calculate the average frequency of outliers (temperature variation exceeding of the set value $\Delta T$ ). All processed records are very well described by the Gaussian distribution:

$v=(0.79 \pm 0.17) \exp \left(-(10.61 \pm 4.5)(\Delta T)^{2}\right)$,

where the parameters vary from station to station. The dimension of $v$ is $\mathrm{h}^{-1}$ and $\Delta T$ is in centigrade. It should be noted that the deviation in $v_{0}$ consists of 21 and $42 \%$ in the exponent, over a study site of $48000 \mathrm{~km}^{2}$ in the tropical part of eastern Atlantic. More details of the experiment data are given in Morozov et al. (1998).

\subsection{Internal wave heights in the Mediterranean Sea}

Let us discuss internal wave statistics in the seas of low tide where one can expect the universe statistical characteristics over a short period of time without correlation to the phases of the moon. The mechanisms of internal wave generation here can be storms and upwelling as well as the effect of river discharge. We analyzed internal wave observations in one of the Mediterranean regions (the Levantine Sea) during the 27th cruise of the RV Professor Kolesnikov (JulyAugust 1991). These old data briefly presented in Ivanov et
Table 2. Predicted internal wave heights in the Mediterranean Sea.

\begin{tabular}{lrrrr}
\hline Time period & 1 day & 1 week & 1 month & 3 months \\
\hline$A(\mathrm{~m})$ & 2.6 & 3.6 & 4.5 & 5.1 \\
\hline
\end{tabular}

al. (1993a) have been revised. During the period from 27 to 29 July 1991 a special experiment to record internal waves was performed in the study site near the Egyptian shelf. A distributed temperature sensor $25 \mathrm{~m}$ long (MHI 4106) was towed in the thermocline along the tacks located as a star. The temperature data were later recalculated into the vertical isopycnal displacement. The basin depth in the study site varies from 200 to $1100 \mathrm{~m}$. The vertical profile of the Brunt-Väisälä frequency contained in the pycnocline presence at a depth of about $25 \mathrm{~m}$ is characterized by a frequency of $17 \mathrm{cycle} \mathrm{h}^{-1}$. Below the pycnocline the mean value of the Brunt-Väisälä frequency is 4 cycle $^{-1}$. The wave height distribution function has been calculated from these data. The vessel speed was approximately $V=5 \mathrm{kn}$, which significantly exceeds the internal wave propagation speed in this region $(c=2 \mathrm{kn})$. Therefore, in the first approximation, the internal wave field can be considered frozen. This means that the "true" time recording can be increased by $V / c=2.5$ times. The applicability of the Gaussian (red) and Poisson (blue) laws for the exceedance (cumulative) frequency, as one can see from Fig. 1, is well applied for the observed data: they are approximated by the formulas

$v=4 \exp (-1.9 A)$

$v=2 \exp \left(-A^{2}\right)$,

where $A$ is a wave amplitude measured in meters, and $v$ is in $\mathrm{h}^{-1}$. Distribution (20) can be used for the prediction of relatively large-amplitude waves. The predicted values of internal wave amplitudes calculated using formula (20a) that can occur in the Mediterranean Sea near the Egyptian shelf over different return periods are summarized in Table 2. Return period is the time period when the wave of predicted amplitude appears at least once.

It should be noted that the observed internal waves in this region have much smaller amplitudes than over the ridges, for example the Mascarene Ridge (Morozov et al., 1996) and in the Luzon Strait, where $100 \mathrm{~m}$ waves are recorded (Ramp et al., 2004). This fact is well known in the seas with low tides and is reflected in the large value of the return period for internal wave of $5 \mathrm{~m}$ amplitude in this part of the Mediterranean Sea. Hence the observed height distribution 


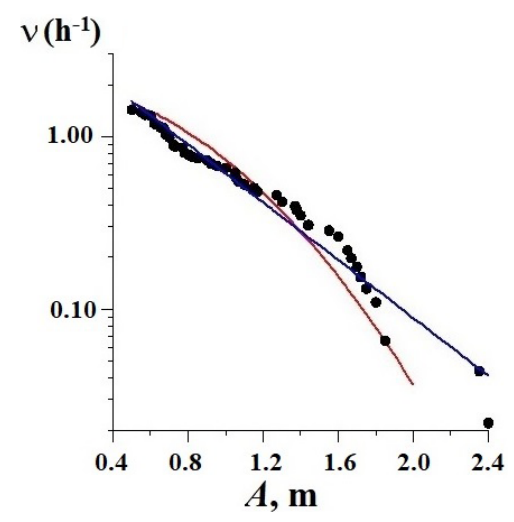

Figure 1. The exceedance frequency of internal wave amplitudes in the eastern part of the Mediterranean Sea.

is in the "middle" between the Gaussian statistics (for weakamplitude waves) and Poisson statistics (for large-amplitude waves).

\subsection{Exceedance frequency in the current velocity from the mooring data (northwestern shelf of Australia)}

Relatively long internal wave records were obtained from moorings on the northwestern shelf of Australia (Pelinovsky et al., 1995). The water depth is approximately $123 \mathrm{~m}$. We shall analyze the velocities in the internal wave range, recorded at a level of $3 \mathrm{~m}$ above the ocean bottom. The time sampling was $2 \mathrm{~min}$, and the duration of measurements was 10 days. Only the velocity component, which contains the strongest wave fluctuations, was analyzed in the transverse to the isobath direction $\left(45^{\circ}\right.$ northeast). The time series were processed by a high-frequency filter to remove the tidal component. Each record was divided into equal intervals of $4000 \mathrm{~min}$. The analysis of time series processing results is reported by Pelinovsky et al. (1995). The calculated values of exceedance frequency for different amplitudes are approximated by the expression

$v=1.33 \exp (-0.071 U)$,

where the dimension of $v$ is $\mathrm{h}^{-1}$ and the dimension of the amplitude of horizontal velocity variation $U$ is in $\mathrm{cm} \mathrm{s}^{-1}$.

The regression formulae presented above can be used to calculate the exceedance probability of large-amplitude internal waves as a function of the amplitude of velocity caused by internal waves and time duration. The results of calculation for the northwestern shelf of Australia are shown in Fig. 2.

\section{Discussion and conclusion}

We have considered statistical characteristics of the internal wave field in several zones of the World Ocean: the tropical part of the western Atlantic Ocean near the mouth of the

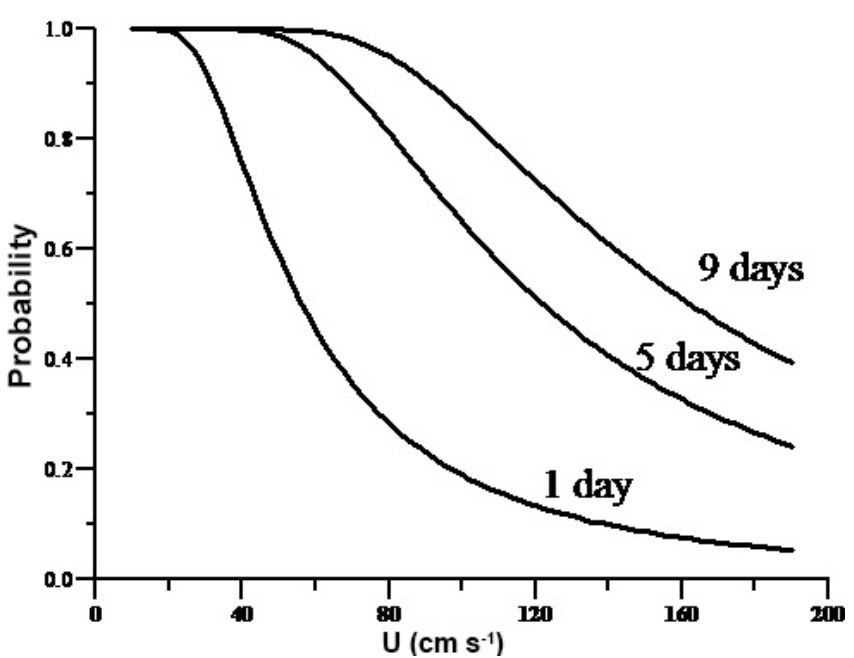

Figure 2. Probability of occurrence of internal waves at the northwestern shelf of Australia.

Amazon, the part of the eastern Atlantic, the western part of the Mediterranean Sea, the northwestern shelf of Australia and the Yellow Sea shelf (Fig. 3).

It is difficult to compare directly the results of exceedance frequency calculations for various regions of the World Ocean. The observations were not performed using similar methods. One of the difficulties is that different characteristics were measured. In particular, in the tropical zone of the Atlantic, the vertical displacement of the sound-scattering layers was measured; in the Mediterranean Sea it was the amplitude of displacement of the thermocline, while on the Australian shelf the records of flow velocity fluctuations were analyzed. At the Mesopolygon- 85 it was the temperature fluctuations. To recalculate these values into the amplitude of internal wave displacement we should know additional information such as the temperature gradient. The next difficulty is that all measurements were produced at different levels. The internal mode structures and hydrology were never analyzed in these measurements, and we cannot say what value of the internal wave amplitude we can expect at the comparison level. So, now we can predict the internal wave amplitude only at the level of measurements.

We find that the Poisson law is valid for internal wave amplitude distribution at the three study sites, but in the Mediterranean, where the internal wave amplitudes did not exceed $2 \mathrm{~m}$, we find that the Gaussian distribution is also appropriate here as the Poisson distribution. The Gaussian law is valid for small amplitudes, and we also obtain this law in Mesopolygon-85.

Meanwhile, the value of $v_{0}$ has universal character and should not depend on the measured characteristics of internal waves. In the Australian shelf $v_{0}=1.3 \mathrm{~h}^{-1}$, in the tropical zone of the Atlantic $v_{0}=9.2 \mathrm{~h}^{-1}$, in Mesopolygon-85 $v_{0}$ lies between 0.62 and $0.96 \mathrm{~h}^{-1}$ and in the Mediterranean (Levantine Sea) $\nu_{0}=4 \mathrm{~h}^{-1}$. In fact the $T_{0}=2 \pi / \nu_{0}$ can be 


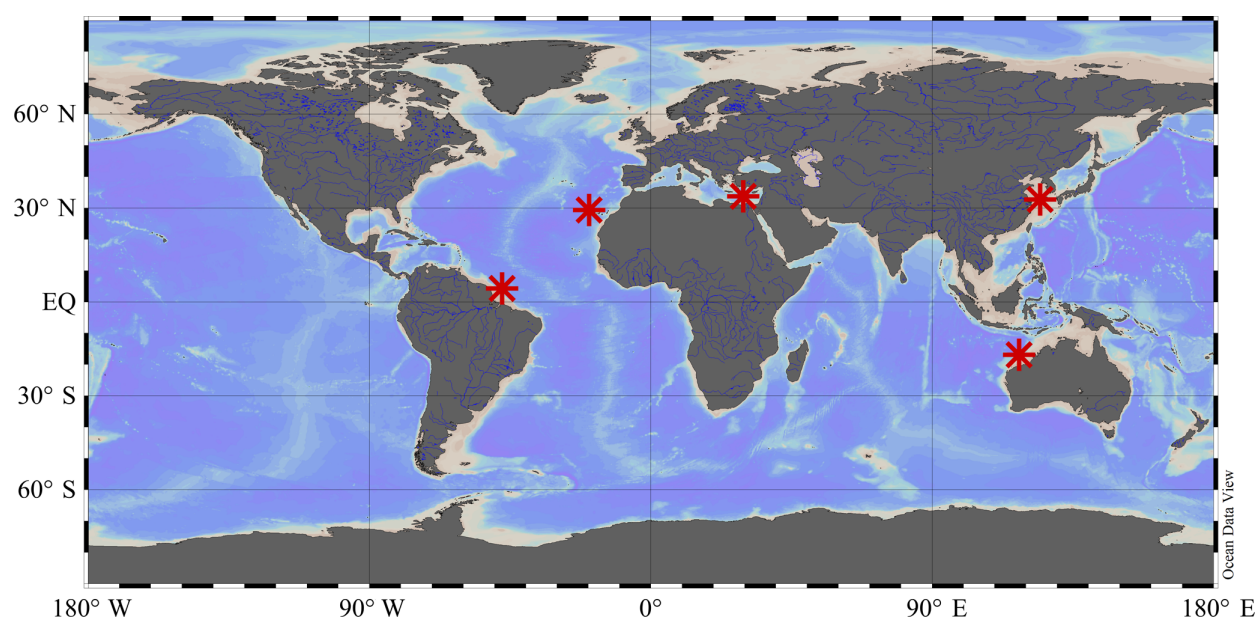

Figure 3. Areas where we consider statistical characteristics of internal waves.

interpreted as the mean time of the internal wave appearance in the study site. Each value is characteristic of the specific ocean region. The scatter of these values is sufficiently high. So, in Mesopolygon- 85 the value of $T_{0}$ lies between 6.5 and $10.1 \mathrm{~h}$. In the Australian shelf the semidiurnal tide is the main factor of the internal wave generation, and $T_{0}$ is about $5 \mathrm{~h}$ here. The time periods of 6.5 and $5 \mathrm{~h}$ are close to the time period of maximal tidal-ebb flow in zone of internal wave generation. The Levantine Sea into the Mediterranean is very intensive in the wave generation, $T_{0}=1.6 \mathrm{~h}$. The tide is not the main reason for internal wave generation here and in the tropical zone of the Atlantic $T_{0}=40 \mathrm{~min}$, which means that the internal waves appear here very often, mainly due to their propagation from zones of generation.

Currently, the numerical methods to predict internal wave field characteristics in different regions of the World Ocean are widely applied (Kurkina and Talipova, 2011; Talipova et al., 2014). They demonstrate that such characteristics are very sensitive to the density stratification of the ocean. The influence of variation of water stratification on the internal wave dynamics can be illustrated by the seasonal maps of kinematic parameters of internal waves (Kurkina et al., 2011, 2017a, b). Statistical estimates of internal waves existing in various regions under different background conditions using numerical models can be calculated. The authors have started to do this work, which will be analyzed further.

Data availability. The data used by this study are extracted from the GDEM database.

Author contributions. All authors made the same contribution to this work.
Competing interests. The authors declare that they have no conflict of interest.

Acknowledgements. This study was initiated in the framework of the state task programme in the sphere of scientific activity of the Ministry of Education and Science of the Russian Federation (project nos. 5.4568.2017/6.7 and 5.1246.2017/4.6) and financially supported by this programme, grants of the President of the Russian Federation (NSh-2685.2018.5 and MK-1124.2018.5) and Russian Foundation for Basic Research (grant no. 16-05-00049).

Authors thank Eugene Morozov and Yury Stepanyants for useful critical comments.

Edited by: Kateryna Terletska

Reviewed by: Eugene Morozov and Yury Stepanyants

\section{References}

Alford, M. H., Peacock, T., MacKinnon, J. A., Nash, J. D., Buijsman, M. C., Centurioni, L. R., Chao, S. Y., Chang, M. H., Farmer, D. M., Fringer, O. B., Fu, K. H., Gallacher, P. C., Graber, H. C., Helfrich, K. R., Jachec, S. M., Jackson, C. R., Klymak, J. M., Ko, D. S., Jan, S., Johnston, T. M. S., Legg, S., Lee, I. H., Lien, R. C., Mercier, M. J., Moum, J. N., Musgrave, R., Park, J. H., Pickering, A. I., Pinkel, R., Rainville, L., Ramp, S. R., Rudnick, D. L., Sarkar, S., Scotti, A., Simmons, H. L., St Laurent, L. C., Venayagamoorthy, S. K., Wang, Y. H., Wang, J., Yang, Y. J., Paluszkiewicz, T., and Tang, T. Y.: The formation and fate of internal waves in the South China Sea, Nature, 521, 65-69, 2015.

Apel, J. R., Holbrock, J. R., Kiu, A. K., and Tsai, J. J.: The Sulu sea internal soliton experiment, J. Phys. Oceanogr., 15, 1625-1651, 1985.

Dischel, R. S.: Climate Risk and the Weather Market, London, Risk Waters Group Ltd, 300 pp., 2002.

Fraser, N.: Surfing an oil rig, Energy Rev., 4, 20, 1999. 
Grimshaw, R., Pelinovsky, E., and Talipova, T.: Modeling internal solitary waves in the coastal ocean, Surv. Geophys., 28, 273-298, 2007.

Gumbel, E. J.: Statistics of extremes, Columbia Univ. Press, New York, 375 pp., 1958.

Helfrich, K. R. and Melville, W. K.: Long nonlinear internal waves, Annu. Rev. Fluid Mech., 38, 395-425, 2006.

Holloway, P., Pelinovsky, E., and Talipova, T.: A generalised Korteweg-de Vries model of internal tide transformation in the coastal zone, J. Geophys. Res., 104, 18333-18350, 1999.

Ivanov, V. A., Pelinovsky, E. N., and Talipova, T. G.: Recurrence frequency of internal wave amplitudes in the Mediterranean, Oceanology, 33, 180-184, 1993a.

Ivanov, V. A., Pelinovsky, E. N., and Talipova, T. G.: The long-time prediction of intense internal wave heights in the tropical region of Atlantic, J. Phys. Oceanography, 23, 2136-2142, 1993 b.

Kaistrenko, V.: Tsunami, Recurrence function: structure, methods of creation, and application for tsunami hazard estimates, Pure Appl. Geophys., 171, 3527-3538, 2014.

Kakutani, T. and Yamasaki, N.: Solitary waves on a two-layer fluid, J. Phys. Soc. Jpn., 45, 674-679, 1978.

Kharif, Ch., Pelinovsky, E., and Slunyaev, A.: Rogue waves in the ocean, Springer, 216 pp., 2009.

Kort, V. G. (Ed.): Hydrophysical studies on the program "Mesopolygon", Nauka, 256 pp., 1988.

Kozlov, I., Romanenkov, D., Zimin, A., and Chapron, B.: SAR observing large-scale nonlinear internal waves in the White Sea, Remote Sens. Environ., 147, 99-107, 2014.

Kurkina, O. E. and Talipova, T. G.: Huge internal waves in the vicinity of the Spitsbergen Island (Barents Sea), Nat. Hazards Earth Syst. Sci., 11, 981-986, https://doi.org/10.5194/nhess-11981-2011, 2011.

Kurkina, O., Talipova, T., Pelinovsky, E., and Soomere, T.: Mapping the internal wave field in the Baltic Sea in the context of sediment transport in shallow water, J. Coast. Res., 64, 2042-2047, 2011.

Kurkina, O., Rouvinskaya, E., Talipova, T., and Soomere, T.: Propagation regimes and populations of internal waves in the Mediterranean Sea basin, Estuar. Coast. Shelf Sci., 185, 44-54, 2017a.

Kurkina, O., Talipova, T. , Soomere, T., Kurkin, A., and Rybin, A.: The impact of seasonal changes in stratification on the dynamics of internal waves in the Sea of Okhotsk, Est. J. Earth Sci., 66, 238-255, 2017b.

Leadbetter, M. R., Lindgren, G., and Rootzen, H.: Extremes and related properties of random sequences and processes, Springer, 336 pp., 1983.

Miropolsky, Yu. Z.: Dynamics of internal gravity waves in the ocean, edited by: Shishkina O., Springer, 321 pp., 2001.

Morozov, E. G.: Semidiurnal internal wave global field, Deep Sea Res., 42, 135-148, 1995.

Morozov, E. G. and Vlasenko, V. I.: Extreme tidal internal waves near the Mascarene Ridge, J. Mar. Syst., 9, 203-210, 1996.

Morozov, E., Pelinovsky, E., and Talipova, T.: Exceedance frequency for internal waves during the Mesopolygon- 85 experiment in the Atlantic, Oceanology, 38, 470-475, 1998.

Morozov, E. G., Trulsen, K., Velarde, M. G., and Vlasenko, V. I., Internal tides in the Strait of Gibraltar, J. Phys. Oceanogr., 32, 3193-3206, 2002.
Morozov, E. G., Parrilla-Barrera, G., Velarde, M. G., and Scherbinin, A. D.: The Straits of Gibraltar and Kara Gates: A Comparison of Internal Tides, Oceanol. Ac., 26, 231-241, 2003.

Morozov, E. G., Paka, V. T., Bakhanov, V. V.: Strong internal tides in the Kara Gates Strait, Geophys. Res. Lett., 35, L16603, https://doi.org/10.1029/2008GL033804, 2008.

Morozov, E. G.: Oceanic internal tides, observations, analysis, and modeling. A global view, Springer, 291 pp., 2018.

Osborne, A.: Nonlinear ocean waves and the inverse scattering transform, Academic Press, 944, 2010.

Pelinovsky, E., Holloway, T., and Talipova, T.: A statistical analysis of extreme events in current variations due to internal waves from the Australian North West Shelf, J. Geophys. Res., 100, 2483124839, 1995.

Pelinovsky, E. and Kharif, C. (Eds.): Extreme ocean waves, 2nd Edition, Springer, 236 pp., https://doi.org/10.1007/978-3-31921575-4, 2016.

Pelinovsky, E. and Shurgalina, E.: KDV soliton gas: interactions and turbulence. Book: Challenges in complexity: dynamics, patterns, cognition, edited by: Aronson, I., Rulkov, N., Pikovsky, A., and Tsimring, L., Series: Nonlinear Systems and Complexity, Springer, 20, 295-306, 2017.

Ramp, S. R., Tang, T. Y., Duda, T. F., Lynch, J. F., Liu, A. K., Chiu, C. S., Bahr, F. L., Kim, H. R., and Yang, Y. J.: Internal solitons in the northeastern South China Sea - Part I: Sources and deep water propagation, IEEE J. Ocean. Eng., 29, 1157-1181, 2004.

Rutenko, A. N.: The influence of internal waves on losses during sound propagation on a shelf, Acoust. Phys., 56, 703-713, 2010.

Sabinin, K. D. and Serebryany, A. N.: "Hot spots" in the field of internal waves in the ocean, Acoust. Phys., 53, 357-380, 2007.

Salusti, F., Lascaratos, A., and Nittis, K.: Changes of polarity in marine internal waves: Field evidence in eastern Mediterranean Sea, Ocean Model., 82, 10-11, 1989.

Shroyer, E. L., Moum, J. N., and Nash, J. D.: Nonlinear internal waves over New Jersey's continental shelf, J. Geoph. Res., 116, C03022, https://doi.org/10.1029/2010JC006332, 2011.

Si, Z., Zhang, Y., and Fan, Z.: A numerical simulation of shear forces and torques exerted by large-amplitude internal solitary waves on a rigid pile in South China Sea, Appl. Ocean Res., 37, 127-132, 2012.

Song, Z. J., Teng, B., Gou, Y., Lu, L., Shi, Z. M., Xiao, Y., and $\mathrm{Qu}, \mathrm{Y}$.: Comparisons of internal solitary wave and surface wave actions on marine structures and their responses, Appl. Ocean Res., 33, 120-129, 2011.

Stöber, U. and Moum, J. N.: On the potential for automated realtime detection of nonlinear internal waves from seafloor pressure measurements, Appl. Ocean Res., 33, 275-285, 2011.

Stuart, C.: An introduction to statistical modeling of extreme values, Springer, 242 pp., 2001.

Talipova, T. G., Kurkina, O. E., Terletska, E. V., Kurkin, A. A., and Rouvinskaya, E. A.: Modeling of internal wave field in the coastal zone of the Barents Sea, Ecol. Syst. Dev., 3, 26-38, 2014.

$\mathrm{Xu}$, J., Chen, Zh., Xie, J., and Cai, Sh.: On generation and evolution of seaward propagating internal solitary waves in the north western South China Sea, Commun. Nonlinear Sci. Numer. Simulat., 32, 122-136, 2016.

$\mathrm{Xu}, \mathrm{Zh}$. and Yin, B.: Variability of internal solitary waves in the Northwest South China Sea, Oceanography, edited by: Marcelli, M., InTech., 131-146, 2012. 
Vlasenko, V., Stashchuk, N., and Hutter, K.: Baroclinic tides: theoretical modeling and observational evidence, Cambridge University Press, 351 pp., 2005.

Wang, T. and Gao, T.: Statistical properties of high-frequency internal waves in Qingdao offshore area of the Yellow Sea, Chinese J. Oceanol. Limnol., 20, 16-21, 2002.

Warn-Varnas, A. C., Chin-Bing, S. A., King, D. B., Hallock, Z., and Hawkins, J. A.: Ocean-acoustic solitary wave studies and predictions, Surv. Geophys., 24, 39-79, 2003.
Zheng, Q., Susanto, R. D., Ho, Ch.-R., Song, Y. T., and $\mathrm{Xu}$, Q.: Statistical and dynamical analyses of generation mechanisms of solitary internal waves in the northern South China Sea, J. Geophys. Res., 112, C03021, https://doi.org/10.1029/2006JC003551, 2007. 